\title{
Effects of Adjunctive Daily Blue Light Toothbrushing on Dental Plaque and Gingival Inflammation-A Randomized Controlled Study
}

\author{
Nadja Bjurshammar¹, Sebastian Malmqvist1, Gunnar Johannsen'1, Elisabeth Boström1, \\ Jonas Fyrestam², Conny Östman³, Annsofi Johannsen ${ }^{1}$ \\ ${ }^{1}$ Department of Dental Medicine, Division of Oral Diseases, Karolinska Institutet, Huddinge, Sweden \\ ${ }^{2}$ Department of Chemistry-BMC, Uppsala University, Uppsala, Sweden \\ ${ }^{3}$ Department of Environmental Science \& Analytical Chemistry, Division of Analytical Chemistry, Stockholm University, \\ Stockholm, Sweden \\ Email: Nadja.Bjurshammar@ki.se
}

How to cite this paper: Bjurshammar, N., Malmqvist, S., Johannsen, G., Boström, E., Fyrestam, J., Östman, C. and Johannsen, A. (2018) Effects of Adjunctive Daily Blue Light Toothbrushing on Dental Plaque and Gingival Inflammation-A Randomized Controlled Study. Open Journal of Stomatology, 8, 287-303.

https://doi.org/10.4236/ojst.2018.810027

Received: August 12, 2018

Accepted: October 21, 2018

Published: October 24, 2018

Copyright $\odot 2018$ by authors and Scientific Research Publishing Inc. This work is licensed under the Creative Commons Attribution International License (CC BY 4.0).

http://creativecommons.org/licenses/by/4.0/

\begin{abstract}
Phototherapy with blue light emitting diodes (LEDs) is gaining interest because of the efficient antimicrobial benefits reported in several studies in the last decade. The aim in this study was to investigate if a toothbrush with incorporated blue light used in daily oral care can reduce dental plaque and gingival inflammation. An 8-week single-blinded randomized controlled clinical study including 48 subjects compared effects of toothbrushes with/without $450 \mathrm{~nm}$ blue LED light emission, on clinical parameters (plaque index, gingival index, bleeding on probing), and on inflammatory markers in saliva and gingival crevicular fluid. Significant reductions in dental plaque and gingival inflammation ( $\mathrm{p}<0.001)$, and in some inflammatory markers $(\mathrm{p} \leq 0.05)$, matrix metalloproteinase (MMP)-8, tissue inhibitor metalloproteinase inhibitor (TIMP)-1, interleukin (IL)- $1 \beta$ and interleukin (IL)-8, were detected within both groups from baseline to follow-up. For all subjects dental plaque was reduced with $57 \%$, and a reduction in gingival inflammation was demonstrated by a decrease in gingival index (GI) with $46 \%$ and in bleeding on probing (BOP) with a decrease of $15 \%$. No significant differences were found between the groups at a level of $p=0.05$. However, the amount of plaque was reduced by $62 \%$ in the blue light group and $51 \%$ in the control group, a difference established at a level of $\mathrm{p}=0.058$. A toothbrush with a $450 \mathrm{~nm}$ LED did not show any statistical significant adjunctive effect of toothbrushing regarding reduction in measurements of dental plaque and gingival inflammation.
\end{abstract}




\section{Keywords}

Phototherapy, Antimicrobial Blue Light, Dental Plaque, Gingival

Inflammation, Toothbrushing

\section{Introduction}

One of the most common infectious diseases is periodontal disease, i.e. gingivitis and periodontitis, where the most prevalent are plaque-induced gingivitis and chronic periodontitis [1]. Epidemiological studies show that gingivitis is globally common in children and adolescents and periodontal destructive disease is found in $15 \%-20 \%$ of middle-aged adults [2] [3]. Poor oral hygiene can trigger an inflammatory response [4], therefore daily oral care is crucial for reducing dental plaque and maintaining a healthy gingivae [5]. Studies have shown that persistent gingival inflammation is a risk factor for developing periodontitis [6] [7] [8]. Thus, it is of a great interest to prevent and treat gingival inflammation and develop effective therapies to control microbial biofilms, i.e. dental plaque. The increasing number of antibiotic resistant strains of microorganisms makes it even more important to develop antibiotic-free alternative treatments [9] [10] [11]. In a review, it is stated that antimicrobial phototherapy with short, daily exposures to blue light is a new research path for prophylaxis in oral hygiene that may have an impact on gingivitis and periodontitis [12]. A broad spectrum of light with wavelengths ranging from $380-520 \mathrm{~nm}$ could potentially give a threefold reduction of especially black pigmented bacteria such as Porphyromonas gingivalis and Prevotella spp. Antimicrobial phototherapy with blue light has two advantages: no chemicals are needed and blue light is regarded safe to use for short-term treatment [13] [14] [15], since exposing mammalian cells with a bactericidal dose $\left(36 \mathrm{~J} / \mathrm{cm}^{2}\right)$ do not cause any damage [16]. Several bacteria that are associated with periodontal disease have been shown to produce endogenous porphyrins [17] [18] [19] [20]. In the presence of light and oxygen, these naturally occurring endogenous porphyrins can act as photosensitizers inside bacteria. Porphyrins have a specific band of absorbance where they can absorb photons and go into a higher energetic state. The excited porphyrin can either return to its ground state by emitting a photon as fluorescence or by intersystem crossing go to an excited triplet state. In this state the energy can be transferred to an oxygen molecule creating singlet oxygen which is highly reactive and can oxidize biomolecules, leading to cytotoxicity. It has been suggested that singlet oxygen is the main active molecular species in phototherapy. In vitro experiments have shown that phototherapy has the ability to inactivate many species of microorganisms, without the addition of an exogenous photosensitizer, by using wavelengths targeting the absorbance of endogenous porphyrin molecules [19] [21] [22] [23] [24] [25]. An in vivo study has shown that when illuminating with blue light at $455 \mathrm{~nm}$ for two minutes, using a light device at a clinic twice daily over a period of four days, the proportion of oral bacteria associated with periodontal 
disease was reduced [26]. Recently, toothbrushes with incorporated blue light have been introduced to the market. However, which wavelength is the most effective has not been established. A pilot clinical study has reported that a blue light-emitting toothbrush with a central wavelength of $412 \mathrm{~nm}$ was significantly more effective compared to a standard toothbrush for the reduction of dental plaque and gingival inflammation [27]. The aim of the present study was to investigate if toothbrushes with incorporated $450 \mathrm{~nm}$ LEDs can reduce dental plaque and gingival inflammation when used in daily oral care.

\section{Materials and Methods}

\subsection{Study Design}

This clinical study was designed as an 8-week single-blinded randomized controlled clinical study comparing toothbrushes with an incorporated $450 \mathrm{~nm}$ blue light LED and toothbrushes without light. The study population was divided into two intervention groups (Group A/C) and two control groups (Group B/D). The analysis followed a per-protocol-analysis concept that better reflected the effects of the treatment with a blue light toothbrush. This analysis was only restricted to the subjects who fulfilled the protocol in the terms of the eligibility, interventions, and outcome assessment.

\subsection{Outcomes}

Expected primary outcomes include: 1) reductions in dental plaque and 2) gingival inflammation from baseline to follow-up visit at week 8. In addition 3) reduction of gingival inflammation when using the two kinds of toothbrushes together with a peroxide compared to a non-peroxide toothpaste. The amount of dental plaque was measured using Plaque Index (PI) [28], while gingival inflammation was assessed using Gingival Index (GI) [29]. Bleeding on Probing Index (BOP), and analyzing inflammatory markers determined by bioanalytical methods were also used for the evaluation.

\subsection{Study Population}

All first year students $(\mathrm{n}=90)$ attending the Dental Hygiene Program at the Department of Dental Medicine, Karolinska Institutet, Stockholm, Sweden, in the autumn semesters of 2014 and 2015 were asked to participate in the clinical study and 68 of the students accepted to participate, of which 61 subjects met the inclusion criteria. Inclusion criteria were the presence of gingival inflammation defined by a relative BOP level above $20 \%$. Exclusion criteria for all subjects were: having periodontitis or any systemic diseases, having used antibiotics and/or anti-inflammatory drugs less than three months prior to the investigation or/and being a current smoker. The students received verbal and written information regarding the study purpose and structure, and a written informed consent from each subject were obtained before commencing the investigations. Throughout the recruitment process it was emphasized that participation in the study was voluntary and that the students were free to withdraw at any time. 
Ethical approval was obtained from the Stockholm Regional Ethical Review Board (Dnr: 2013/200-31/3, 2014/1197-32, 2016/2348-31).

\subsection{Randomization and Blinding}

The non-blinded research leader (GJ) was responsible for the randomization process, as well as for distributing toothbrushes (with or without blue light) and toothpastes (with or without peroxide) to the subjects. Alphabetical lists of the eligible subjects were used by the research leader to assign the subjects with an odd ranking number on the alphabetical list to a control group, and subjects with an even number to an intervention group. The lists of the assignment of the participants were kept by the research leader until the end of the study. In 2014 both groups were given the same gel toothpaste and the intervention group used a $450 \mathrm{~nm}$ blue LED light toothbrush (Group A), while the control group used a toothbrush with the blue light disabled (Group B). In 2015 every second subject in each group used a toothpaste containing peroxide and the intervention group used a $450 \mathrm{~nm}$ blue LED light toothbrush (Group C), while the control group used a toothbrush with the blue light disabled (Group D). The subjects could for an obvious reason not be blinded as to which toothbrush they were using since one toothbrush was emitting blue light while the other was not. The clinical examination was performed by three experienced clinicians of which one (AJ) was responsible for the sample collection of saliva and gingival crevicular fluid (GCF), while the other two (NB, SM) performed the clinical assessments and were both blinded regarding group assignment. Examiner NB registered both PI and GI at all visits on every subject. Examiner SM performed all measurements of BOP and probing pocket depth (PPD) at baseline as well as at the follow up visit after eight weeks.

\subsection{Toothbrushes}

The intervention groups were given a manual toothbrush incorporated with a light emitting diode (LED) emitting blue light with an emission maximum at 450 $\mathrm{nm}$ and with a power density of $13.5 \mathrm{~mW} / \mathrm{cm}^{2}$ at $5.9 \mathrm{~mm}$ from the end of the bristles $^{1}$. This toothbrush had a built-in 2 minutes timer and the subjects in the intervention groups were informed to brush their teeth until the blue light turned off. The toothbrush contains no harmful ultraviolet (UV) radiation and meets the standards of the Restriction of Hazardous Substances Directive 2002/95/EC [30]. Tests performed by Technical Research Institute of Sweden (SP) have shown that a $450 \mathrm{~nm}$ LED as the one used in this toothbrush is free from harmful UV radiation [31]. The control groups received the same kind of toothbrush with the light option disabled. The control groups were informed to brush 2 minutes twice a day, i.e. the same time as the intervention groups.

\subsection{Toothpastes}

As described above both the intervention groups $(\mathrm{A} / \mathrm{C})$ and the control groups ${ }^{1}$ UltraBlu $^{\circledR}$, TJL Enterprises, Long beach, CA, USA. 
(B/D) used two kinds of commercially available toothpastes, a regular fluoride-containing gel toothpaste ${ }^{2}$ and a peroxide toothpaste containing fluoride ${ }^{3}$. The latter has been accepted by the Council on Scientific Affairs, American Dental Association and is considered safe for home-use. According to the manufacturer of the toothbrushes, blue light in combination with peroxide can have an adjunctive antimicrobial effect. The ability of the blue light to pass through the toothpaste was tested. Each toothpaste was dissolved in deionized water and analyzed with an UV-Visible spectrophotometer ${ }^{4}$ scanning from $200-700 \mathrm{~nm}$. None of the toothpastes showed any absorbance peak in the blue light region $(400-500 \mathrm{~nm})$ and was therefore considered not to affect the intensity of the emitted light by absorption.

\subsection{Sampling of Saliva and Gingival Crevicular Fluid}

Collection of unstimulated saliva and gingival crevicular fluid (GCF) was performed at baseline and after 8 weeks. The subjects were requested not to eat or drink anything, except water, 1 hour before saliva collection minimizing possible food debris. All saliva produced during a 15 minutes period was transferred into a test tube. The samples were immediately centrifuged at $1500 \mathrm{rpm}$ for $10 \mathrm{mi}$ nutes at $4^{\circ} \mathrm{C}$. After centrifugation, the supernatant was transferred to five Eppendorf tubes each containing $200 \mu \mathrm{L}$ saliva. The tubes were sealed and stored at $-80^{\circ} \mathrm{C}$ pending analysis. GCF was collected from the same mesiobuccal gingival pockets of one maxillary first molar (14) and one mandibular first molar (19) in each subject. Sampling was performed using a paper strip ${ }^{5}$ which was inserted into the gingival crevice and kept in place for 30 seconds. The paper strip was then retracted and put in a $1 \mathrm{~mL}$ Eppendorf tube containing $300 \mu \mathrm{L}$ phosphate buffer solution. The tube was vortexed for 30 seconds, where after the strip was removed and the tube centrifuged for 5 minutes at $8000 \mathrm{rpm}$. The supernatant was transferred to a new Eppendorf tube which was stored at $-80^{\circ} \mathrm{C}$ until analysis of the content of selected immunological inflammatory markers was made.

\subsection{Clinical Examination and Prophylaxis}

A flow chart of the clinical study's proceedings is shown in Table 1. Two weeks before baseline all subjects received a professional supra-gingival scaling and polishing performed by two examiners (NB, SM). At baseline, all subjects were subjected to a clinical periodontal examination including PI, GI PPD and BOP. These indices were measured at four sites on each tooth, while PPD and BOP were measured at six sites per tooth excluding third molars, using a manual periodontal probe ${ }^{6}$. All subjects were then given oral prophylactic information and instruction in oral hygiene procedures, i.e. to brush twice a day during two ${ }^{2}$ Apoteket AB, Solna, Sweden.

${ }^{3}$ Rembrandt, Deeply White, +Peroxide, Johnson \& Johnson Healthcare Products Division of McNeil-PPC, Inc, Skillman, NJ.

${ }^{4}$ Evolution ${ }^{\mathrm{TM}}$ 60S, ThermoFisher, Waltham, MA, USA.

${ }^{5}$ Periopaper; Oraflow Inc., Planiview, NY, USA.

${ }^{6} \mathrm{Hu}-$ Friedy PQ2N7, Chicago, IL, USA. 
Table 1. Flow chart of the study design.

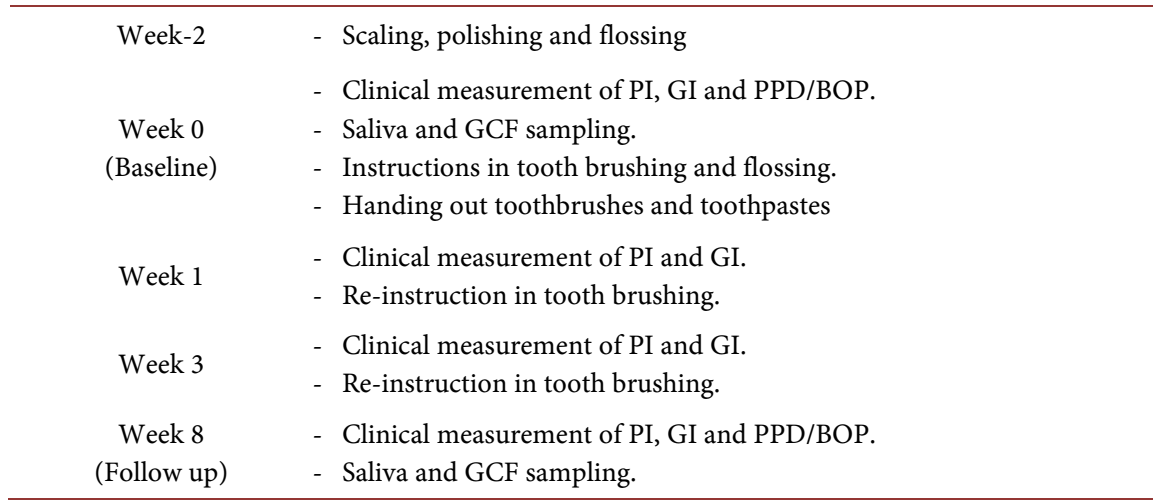

minutes and instruction in flossing technique, as well as to refrain from using antiseptics. After both one and three weeks PI and GI scores were recorded and repeated oral hygiene instructions and a follow-up instruction in oral hygiene were given. After 8 weeks, the same clinical parameters and samples were collected in the same manner as at baseline.

\subsection{Immunological Analysis}

The levels of interleukin-1 $\beta$ (IL-1 $\beta$ ), interleukin-6 (IL-6), interleukin-8 (IL-8), and tumor necrosis factor (TNF- $\alpha$ ) were determined in GCF and saliva by a Luminex bead-based multiplex assay using a High Performance Assay kit ${ }^{7}$ according to the manufacturer instructions on a Bioplex Suspension Array System ${ }^{8}$. The levels of matrix metalloproteinase (MMP-8) and metallopeptidase inhibitor (TIMP-1) in saliva were determined by the Enzyme Linked Immunosorbent Assay (ELISA) ${ }^{9}$. In brief, samples were thawed on ice, diluted, and added with standard concentrations of MMP-8 and TIMP-1 to plates pre-coated with monoclonal antibodies specific for MMP-8 and TIMP-1. After 2 hours of incubation, the plates were washed and antibodies conjugated with horseradish peroxidase were added and incubated for 2 hours. Following washing of the plates, substrate solution was added to generate a color reaction, which was stopped by adding stop solution. The color reactions on the plates were read at $450 \mathrm{~nm}$ using a microplate spectrophotometer ${ }^{10}$.

\subsection{Statistical Analysis}

A power analysis was carried out prior to the study and showed that 48 subjects (24 intervention subjects and 24 control subjects) should be sufficient to detect a difference of $12.5 \%$ between the two groups with a power of $80 \%$ and a two tailed $\alpha$ of $5.0 \%$, with BOP as primary outcome variable. For each patient the average PI and GI indices were calculated as the sum of the individual index for each tooth surface divided with the total number of measured surfaces. The av-

${ }^{7} \mathrm{R}$ \& D Systems Inc; Minneapolis, MN, USA.

${ }^{8}$ Bio-Rad Laboratories, Hercules, CA, USA.

${ }^{9} \mathrm{R} \&$ \& Systems Inc; Minneapolis, MN, USA

${ }^{10}$ SpectraMAX 340, Molecular Devices, Sunnyvale, CA, USA. 
erage PPD was calculated as the sum of the measured PPD for each tooth with $\mathrm{PPD} \geq 4 \mathrm{~mm}$ divided with the number of teeth having a PPD $\geq 4 \mathrm{~mm}$. For BOP the sum of the number of surfaces showing bleeding upon probing was calculated. The change in each index was calculated as:

$$
\text { Absolute index change }=\operatorname{Index}_{\text {Week8 }}-\operatorname{Index}_{\text {Baseline }}
$$

For some statistical analyses a normalized index change was used:

$$
\text { Normalized index change }=\frac{\text { Index }_{\text {Week8 }}-\text { Index }_{\text {Baseline }}}{\text { Index }_{\text {Baseline }}} \times 100
$$

Statistical data analysis was made by using Shapiro-Wilk test for normal distribution, F-test for variance, and t-test for two sample means and paired t-test ${ }^{11}$ to determine the statistical significance in the changes of clinical indices (GI, PI and $\mathrm{BOP}$ ) and in the concentrations of inflammatory markers (MMP-8, TIMP, IL- $1 \beta$, IL-6, IL-8 and TNF- $\alpha$ ).

\section{Results}

\subsection{Subject Sample}

Forty-eight subjects completed the entire clinical trial according to the protocol (twenty-five in the intervention groups, twenty-three in the control groups). There were thirteen drop-outs during the study and the reasons for that was that five subjects dropped out from the Dental Hygiene Program, one were pressed for time, one did not like the taste of the peroxide toothpaste, two experienced tooth hypersensitivity from the Pharmacy toothpaste, two felt the bristles of the toothbrush to hard and two of the drop-outs were of unknown causes.

At baseline, there were no significant differences observed regarding mean age and gender or in clinical indices PI, GI, BOP and PPD between subjects allocated to the control and intervention groups, Table 2. No adverse effects to hard or soft tissues was reported from the subjects or observed by the clinicians.

\subsection{Clinical Assessments}

According to the inclusion criteria, all subjects had a BOP-level above $20 \%$, thus all the subjects entered the study with gingival inflammation.

From baseline to the follow-up visit at week 8 a significant reduction in the average PI, GI and BOP indices was observed for all participants in both groups, Table 3.

Figure 1 shows the average decrease the PI and GI indices for all subjects during the four visits. Both indices showed a statistical significant steady decrease between each occasion during the study. A weak correlation was also found between the normalized decreases in these clinical parameters for all subjects from baseline to week 8 , where GI and BOP showed a weak correlation $\left(\mathrm{R}^{2}\right.$ $=0.31$ ) which both measures gingival inflammation. For PPD as well as for saliva flow no significant change in any of the groups was found during the study.

\footnotetext{
${ }^{11}$ Excel 2010, Microsoft, Redmond, WA, USA.
} 
Table 2. Demographic and clinical data at baseline of subjects that completed the entire study and followed the per-protocol analysis concept.

\begin{tabular}{lccc}
\hline \multirow{2}{*}{ Parameter } & \multicolumn{2}{c}{ Brushing groups } \\
\cline { 2 - 4 } & $\begin{array}{c}\text { Control } \\
\text { (Group B and D) }\end{array}$ & $\begin{array}{c}\text { Intervention } \\
(\text { Group A and C) }\end{array}$ & p-value \\
\hline N & 23 & 25 & \\
Age, mean (SD) & $24.91( \pm 7.15)$ & $23.68( \pm 5.09)$ & 0.50 \\
Gender (male/female) & $4 / 19$ & $2 / 23$ & \\
Baseline data full mouth (excluding 3 $3^{\text {rd }}$ molars) & & & \\
- PI 0 - 3, mean (SD) & $0.49( \pm 0.17)$ & $0.58( \pm 0.27)$ & 0.19 \\
- GI 0 - 3, mean (SD) & $0.65( \pm 0.22)$ & $0.67( \pm 0.23)$ & 0.67 \\
- BOP (\%), mean (SD) & $46.6( \pm 12.8)$ & $52.1( \pm 14.7)$ & 0.19 \\
- number of sites with PD $\geq 4 \mathrm{~mm}$ & 3.74 & 3.08 & \\
- number of sites with PD $\geq 6 \mathrm{~mm}$ & 0.22 & 0.04 & \\
\hline
\end{tabular}

Table 3. Mean values with standard deviation in parentheses for the clinical parameters Plaque Index (PI), Gingival Index (GI), and Bleeding on Probing (BOP). All objects ( $\mathrm{n}=$ $48)$, control groups $(n=23)$ and blue light intervention groups $(n=25)$. $p$-values are given for t-tests, see comments below.

\begin{tabular}{|c|c|c|c|c|c|c|c|c|}
\hline Examination & All & $\mathrm{p}$ & Control & $\mathrm{p}$ & \multicolumn{2}{|c|}{ Intervention } & $\mathrm{p}$ & $\mathrm{p}$ \\
\hline \multicolumn{9}{|c|}{ Plaque Index (PI) } \\
\hline Baseline & $0.54(0.23)$ & - & $0.49(0.17)$ & - & \multicolumn{2}{|c|}{$0.58(0.27)$} & - & $0.19^{c}$ \\
\hline Week 1 & $0.41(0.27)$ & $<0.001^{\mathrm{a}}$ & $0.41(0.26)$ & $0.052^{\mathrm{a}}$ & \multicolumn{2}{|c|}{$0.51(0.26)$} & $0.002^{\mathrm{a}}$ & \\
\hline Week 3 & $0.30(0.16)$ & $<0.001^{\mathrm{a}}$ & $0.29(0.17)$ & $0.006^{\mathrm{a}}$ & \multicolumn{2}{|c|}{$0.30(0.15)$} & $0.055^{\mathrm{a}}$ & \\
\hline Week 8 & $0.23(0.16)$ & $<0.001^{\mathrm{a}}$ & $0.24(0.19)$ & $0.023^{\mathrm{a}}$ & \multicolumn{2}{|c|}{$0.22(0.29)$} & $0.002^{\mathrm{a}}$ & \\
\hline $\mathrm{P}_{\text {Baseline-Week 8 }}$ & \multicolumn{2}{|c|}{$<0.001^{\mathrm{b}}$} & \multicolumn{2}{|c|}{$<0.001^{\mathrm{b}}$} & \multicolumn{3}{|c|}{$<0.001^{\mathrm{b}}$} & $0.058^{\mathrm{d}}$ \\
\hline \multicolumn{9}{|c|}{ Gingival Index (GI) } \\
\hline Baseline & $0.66(0.21)$ & - & $0.65(0.21)$ & - & 0.67 & $(0.23)$ & - & $0.67^{c}$ \\
\hline Week 1 & $0.56(0.17)$ & $<0.001^{\mathrm{a}}$ & $0.55(0.17)$ & $0.028^{\mathrm{a}}$ & 0.57 & $(0.18)$ & $0.009^{\mathrm{a}}$ & \\
\hline Week 3 & $0.46(0.19)$ & $<0.001^{\mathrm{a}}$ & $0.45(0.19)$ & $0.036^{\mathrm{a}}$ & 0.48 & $(0.20)$ & $0.012^{\mathrm{a}}$ & \\
\hline Week 8 & $0.36(0.18)$ & $<0.001^{\mathrm{a}}$ & $0.37(0.18)$ & $0.055^{\mathrm{a}}$ & 0.35 & $(0.18)$ & $<0.001^{\mathrm{a}}$ & \\
\hline $\mathrm{P}_{\text {Baseline-Week 8 }}$ & $<0.0$ & & $<0.00$ & & & $<0.00$ & & $0.39^{\mathrm{d}}$ \\
\hline \multicolumn{9}{|c|}{ Bleeding on Probing Index (BOP) } \\
\hline Baseline & \multicolumn{2}{|c|}{$49.5(14.1)$} & \multicolumn{2}{|c|}{$46.6(12.8)$} & \multicolumn{3}{|c|}{$52.1(14.7)$} & $0.19^{c}$ \\
\hline Week 8 & \multicolumn{2}{|c|}{$42.1(14.9)$} & \multicolumn{2}{|c|}{$41.1(14.7)$} & \multicolumn{3}{|c|}{$43.1(15.1)$} & \\
\hline $\mathrm{P}_{\text {Baseline-Week } 8}$ & \multicolumn{2}{|c|}{$<0.001^{\mathrm{b}}$} & \multicolumn{2}{|c|}{$<0.001^{\mathrm{b}}$} & \multicolumn{3}{|c|}{$<0.001^{\mathrm{b}}$} & $0.29^{\mathrm{d}}$ \\
\hline
\end{tabular}

a) p-value for paired t-test of the decrease in parameters between consecutive examinations; b) p-value for paired t-test of the decrease in parameters between baseline and week 8 ; c) p-value for t-test for difference between control and intervention groups at baseline; $d$ ) p-value for t-test for difference in decrease between control and intervention group from baseline to week 8 .

In Figure 2, as well as in Table 3, the changes in PI, GI and BOP for the control and the intervention groups are shown. When comparing the intervention 


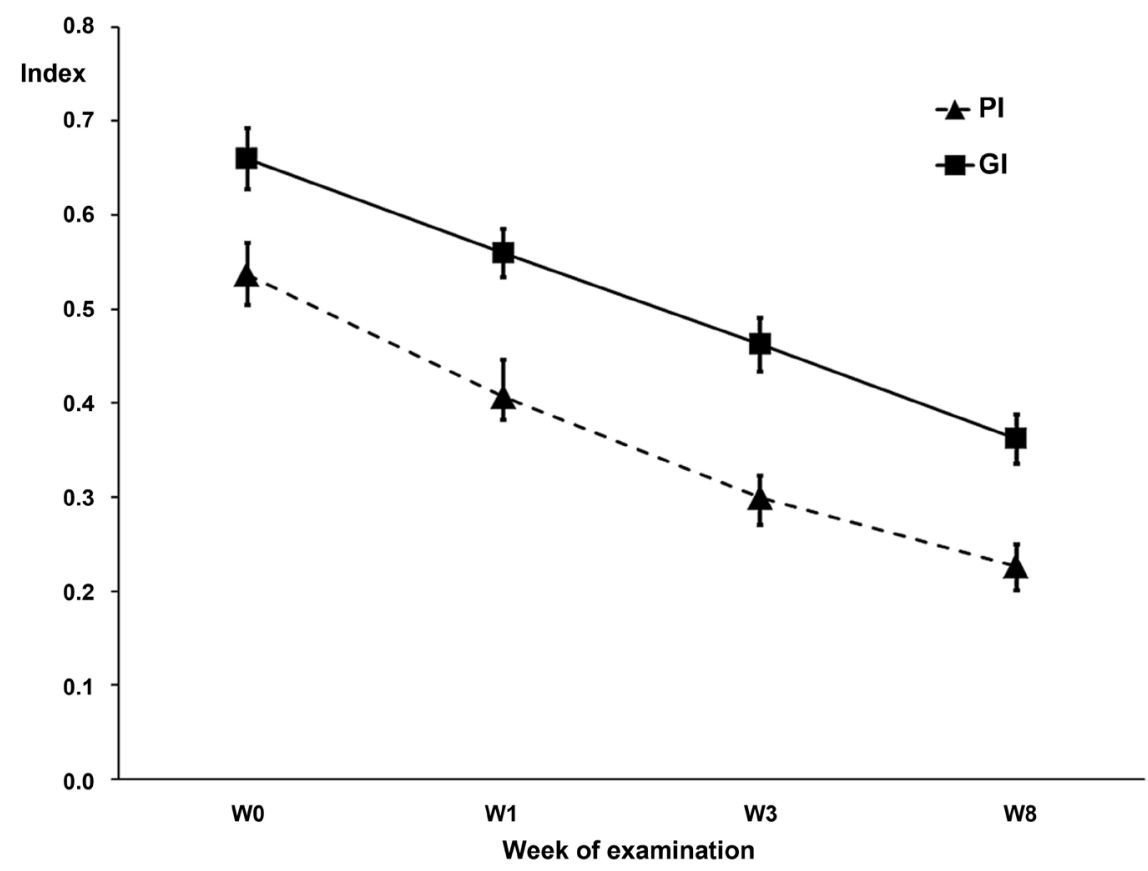

Figure 1. Change in the clinical parameters PI (dashed line) and GI (solid line) for all subjects from Baseline (W0) to Week 8 (W8) plotted with the Standard Error of the Mean as the error bars. The decrease between each week of examinations were all significant with $\mathrm{p}<0.001, \mathrm{n}=48$.

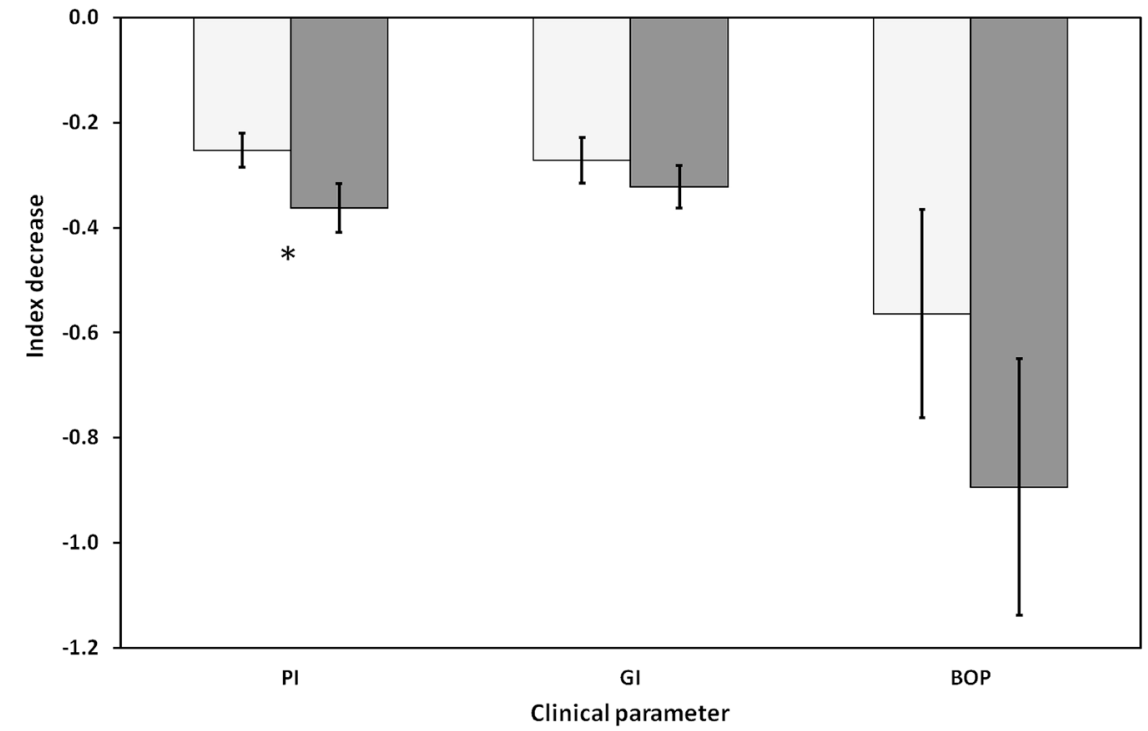

Figure 2. Decrease in the clinical parameters PI, GI and BOP for the control groups (toothbrush without LED light, [light grey]) and the intervention groups (toothbrush with $450 \mathrm{~nm}$ blue LED light [dark grey]) with the standard error of the mean as the error bars. The bars for BOP have been multiplied with 0.1 in order to obtain a comparable scale in the diagram. ${ }^{\star}=$ difference between groups at $\mathrm{p}=0.058$.

groups $(\mathrm{A} / \mathrm{C})$ and the control groups $(\mathrm{B} / \mathrm{D})$ the decrease in all three indices were larger for the intervention groups using the blue light toothbrush compared to the control groups. 
The difference in the decrease in PI from baseline to the last follow-up visit at week 8 is significant at a level of $p=0.058$, close to accepted level $p=0.05$. For GI and BOP there was no statistical significant difference with $\mathrm{p}=0.39$ and $\mathrm{p}=$ 0.29 respectively.

We also investigated if the effect of blue light on reducing plaque or gingival inflammation could be enhanced by the use of a peroxide containing toothpaste. The statistical analyses of the clinical parameters PI, GI and BOP indices as well as the inflammatory markers showed no differences between the toothpaste with or without peroxide (data not shown).

\subsection{Immunological Assessments}

The levels of MMP-8, TIMP-1, IL- $1 \beta$, IL-6, IL- 8 and TNF- $\alpha$ were determined in both saliva and GCF at baseline and at last follow-up visit at week 8 , Table 4. For IL- 6 and TNF- $\alpha$ in saliva and GCF as well as MMP- 8 and TIMP-1 in GCF, the levels were close to, or below, limit of detection (LOD).

These data were not subject to further statistical analysis. The concentrations of IL- $1 \beta$ and IL- 8 in saliva were well above LOD, but showed dispersive results

Table 4. Mean values with standard deviation in parentheses for the inflammatory markers MMP1 and TIMP1 in saliva, and IL- $1 \beta$ and IL8 in gingival crevicular fluid (GCF) at Baseline and Week 8. $\mathrm{p}$-values given for t-tests, see comments below. $\mathrm{n}=$ number of subjects. Concentrations given in picomolar.

\begin{tabular}{|c|c|c|c|c|c|c|c|}
\hline Examination & All & $\mathrm{n}$ & Control & $\mathrm{n}$ & Intervention & $\mathrm{n}$ & $\mathrm{p}$ \\
\hline \multicolumn{8}{|c|}{ Matrix Metalloproteinase-8 (MMP8) in saliva } \\
\hline Baseline & $46.5(40.2)$ & \multirow[b]{2}{*}{42} & $46.5(37.7)$ & \multirow[b]{2}{*}{18} & $46.5(44.0)$ & \multirow[b]{2}{*}{24} & $0.99^{\mathrm{b}}$ \\
\hline Week 8 & $27.5(28.9)$ & & $26.3(35.2)$ & & $30.8(25.7)$ & & \\
\hline $\mathrm{P}_{\text {Baseline-Week } 8}$ & $0.003^{\mathrm{a}}$ & & $0.042^{\mathrm{a}}$ & & $0.029^{\mathrm{a}}$ & & $0.54^{\mathrm{c}}$ \\
\hline \multicolumn{8}{|c|}{ Metallopeptidase Inhibitor 1 (TIMP1) in saliva } \\
\hline Baseline & $263(118)$ & \multirow[b]{2}{*}{47} & $270(128)$ & \multirow[b]{2}{*}{22} & 237 (119) & \multirow[b]{2}{*}{25} & $0.72^{\mathrm{b}}$ \\
\hline Week 8 & $225(111)$ & & $257(110)$ & & $214(104)$ & & \\
\hline $\mathrm{P}_{\text {Baseline-Week } 8}$ & $0.002^{\mathrm{a}}$ & & $0.064^{\mathrm{a}}$ & & $0.008^{\mathrm{a}}$ & & $0.58^{\mathrm{c}}$ \\
\hline \multicolumn{8}{|c|}{ Interleukin $1 \beta(\operatorname{IL} 1 \beta)$ in GCF } \\
\hline Baseline & $106(143)$ & \multirow[b]{2}{*}{48} & $85.7(83.4)$ & \multirow[b]{2}{*}{23} & $125(182)$ & \multirow[b]{2}{*}{25} & $0.34^{\mathrm{b}}$ \\
\hline Week 8 & $46.9(53.2)$ & & $56.2(62.5)$ & & $38.3(42.4)$ & & \\
\hline $\mathrm{P}_{\text {Baseline-Week } 8}$ & $0.009^{\mathrm{a}}$ & & $0.13^{\mathrm{a}}$ & & $0.029^{\mathrm{a}}$ & & $0.44^{\mathrm{c}}$ \\
\hline \multicolumn{8}{|c|}{ Interleukin $1 \beta(\operatorname{IL} 1 \beta)$ in GCF } \\
\hline Baseline & $325(240)$ & \multirow[b]{2}{*}{46} & $310(266)$ & \multirow[b]{2}{*}{22} & $338(217)$ & \multirow[b]{2}{*}{24} & $0.72^{\mathrm{b}}$ \\
\hline Week 8 & $181(142)$ & & $201(152)$ & & $163(133)$ & & \\
\hline $\mathrm{P}_{\text {Baseline-Week } 8}$ & $0.002^{\mathrm{a}}$ & & $0.12^{\mathrm{a}}$ & & $0.002^{\mathrm{a}}$ & & $0.45^{\mathrm{c}}$ \\
\hline
\end{tabular}

a) p-value for paired t-test of the decrease in parameters between baseline and week 8 ; b) p-value for t-test for difference between control and intervention groups at baseline; c) p-value for pt-test for difference in decrease between control and intervention groups from baseline to week 8 . 
making also these data not useful for further statistical analysis. Thus, the inflammatory markers that were further analyzed were MMP-8 and TIMP- 1 in saliva, and IL- $1 \beta$ and IL- 8 in GCF.

When looking at all the subjects, salivary MMP- 8 and TIMP- 1 as well as IL- $1 \beta$ and IL-8 in GCF decreased significantly $(\mathrm{p}<0.01)$ from baseline to the final follow-up visit at week 8 , Table 4 . When comparing the intervention $(\mathrm{A} / \mathrm{C})$ and the control (B/D) groups, no significant difference ( $\mathrm{p} \geq 0.44)$ could be detected between the groups. However, an interesting observation was that there were significant decreases in the salivary levels of both MMP-8 $(p=0.029)$ and TIMP-1 $(\mathrm{p}=0.0073)$, as well as for IL-1 $\beta(\mathrm{p}=0.029)$ and IL-8 $(\mathrm{p}=0.0017)$ in GCF in the blue light toothbrush intervention groups. For the control groups on the other hand, a significant decrease between baseline and week 8 could only be detected for MMP-8 in saliva $(\mathrm{p}=0.042)$. As for the clinical indices, the statistical data analysis of the inflammatory parameters showed no difference when comparing peroxide or non-peroxide toothpastes, with or without blue light illumination (data not shown).

\section{Discussion}

The primary aim of this study was to investigate if a toothbrush with an incorporated LED emitting blue light at $450 \mathrm{~nm}$ has any adjunctive effect on toothbrushing regarding reduction of dental plaque and gingival inflammation.

\subsection{Clinical Indices}

For all subjects in the intervention as well as in the control groups, there were significant decreases in the clinical indices PI, GI and BOP from baseline to the final follow-up visit at week 8 . The average PI for all subjects decreased with $57 \%$ ( $\mathrm{n}=48, \mathrm{p} \leq 0.0001)$, indicating that that the subjects in general had a substantial reduction of the amount of dental plaque. A significant reduction in gingival inflammation was also demonstrated by reductions in both GI and BOP indices, $46 \%$ and $15 \%$ respectively $(\mathrm{n}=48, \mathrm{p} \leq 0.0001)$. A meta-review has assessed 6-month-clinical controlled studies regarding self-performed toothbrushing in adults with gingivitis. Their conclusion was that a single hygiene instruction had a significant positive effect on the reduction of gingival inflammation even though it was small [32]. Improvements in the subjects' oral health status shown in the present study verify these suggestions even though the observations only were conducted for 8 weeks. All subjects in both groups got repeated instructions in toothbrushing at all visits except the final which can explain the significant decreases in all three clinical indices.

However, an interesting observation was made when the blue light intervention groups were compared with the control groups with respect to the clinical indices. All three clinical indices showed a larger decrease in the blue light intervention groups compared to the control. A difference in the PI index decrease could be established at a level of $\mathrm{p}=0.058$, but there was no significant differ- 
ence observed in the GI and BOP indices ( $p>0.29$ ). In a recent study with a similar study design as in the present study, the effect of a blue light-emitting toothbrush with a central wavelength of $412 \mathrm{~nm}$ LED was investigated [27]. After four weeks significant improvements with up to $50 \%$ decrease in plaque and gingival inflammation indices were detected in the treatment group relative to the control group. Their results indicate that the wavelength chosen is of a great importance.

\subsection{Inflammatory Markers}

The levels of IL- $1 \beta$ and IL- 8 in GCF, and MMP- 8 and TIMP-1 in saliva demonstrated a significant decrease when looking at all subjects. The potential of inflammatory markers in GCF and saliva to reflect gingival inflammation has been widely studied, where MMP-8 levels in GCF have been reported to differ between periodontal healthy subjects and subjects with gingivitis and periodontitis [33], putting forward that MMP-8 is a significant marker for determining the degree of inflammation. Some studies have detected elevated concentrations of IL-1 $\beta$, IL-6, and IL-8 associated with experimental gingivitis [34] [35], while other studies have found no differences in the levels of IL- $1 \beta$, as well as MMP-8, in saliva between gingivitis and healthy sites [36]. In a recent review it was concluded that there are sufficient evidence showing that IL- $1 \beta$ in saliva as well as in GCF can be used as markers of the degree of gingival inflammation [37]. In the study presented here, the decrease in the levels of IL- $1 \beta$ and IL- 8 in GCF, and MMP- 8 and TIMP- 1 in saliva were in agreement with the decrease in the GI and BOP clinical indices in all studied objects. However, when comparing the control and blue light intervention groups no differences could be found. Some interesting observations were made. In the blue light intervention groups there were statistical significant decreases in the inflammatory markers IL- $1 \beta$ and IL- 8 in GCF, as well as in MMP-8 and TIMP-1 in saliva from baseline to the final week 8 in the intervention groups. In contrast, the control groups exhibited a statistical significant decrease in the concentration of MMP-8 in saliva. These results imply that there might be a possible adjunct effect of the blue light in combination with tooth brushing evidenced by potential subclinical changes in the levels of the investigated inflammatory markers. Subclinical changes in the IL-1B levels in GCF have previously been shown in response to plaque accumulation prior to clinical signs of inflammation [38]. In the present study, the IL- $1 \beta$ concentration in saliva were well above LOD but showed very divergent results and because of that IL- $1 \beta$ could not be used as a reliable marker.

\subsection{Porphyrins, Blue Light and the LED Toothbrush}

The toothbrush used in this study was the commercially available UltraBlu ${ }^{\circledR}$ which is equipped with a $450 \mathrm{~nm}$ emitting LED. It showed a tendency of positive adjunctive effect to the daily toothbrushing. A toothbrush with a wavelength of $405 \mathrm{~nm}$ should theoretically be more suitable for this kind of adjunctive phototherapy. A $405 \mathrm{~nm}$ version of this toothbrush was under development by the 
manufacturing company, but they were not able to finish it before the end of this project. This was a limitation in this study. A wavelength of $405 \mathrm{~nm}$ has been suggested to be more effective in a number of studies [22] [39] [40], as well as in our in vitro studies of bacteria killing efficiency and porphyrin content [17] [18] [41] [42]. In the latter studies we have shown a connection between the porphyrin content in Escherichia coli and the killing efficiency of this bacterium by blue light at $405 \mathrm{~nm}$. We also showed that at this wavelength, porphyrins are stimulated to produce singlet oxygen.

The proposed mechanism of phototherapy is that endogenous porphyrins, mainly protoporphyrin IX (PPIX), absorb the light and enter into an exited state [23]. The excess energy in the molecule can then be transferred to yield reactive oxygen species (ROS) which are responsible for killing the bacteria. The porphyrins present in oral bacteria are mainly intermediates in the endogenous heme synthesis. These porphyrins all have absorption maxima around $405 \mathrm{~nm}$, while the absorption of light at a wavelength of $450 \mathrm{~nm}$ is much lower [24] [43] [44] [45]. Since the typical bandwidth at 50\% emission level for the LED in the toothbrush used in this study was around $20-30 \mathrm{~nm}$, only a small amount of the light energy can be adsorbed by e.g. PPIX. That $405 \mathrm{~nm}$ should be the optimal wavelength to include in a LED toothbrush is supported by several other studies. In a pilot study our research group has demonstrated efficient inhibition of the growth of $A$. actinomycetemcomitans cultivated on agar plates when illuminated with $405 \mathrm{~nm}$ LED light (unpublished data). In our study there are only weak indications that the $450 \mathrm{~nm}$ toothbrush improves the oral health status of the subjects. The discrepancy between our study and the results in studies by Genina [27] and others [24] [43] [44] [45], is most likely explained by the differences in wavelengths of the LEDs used in the toothbrushes.

\section{Conclusion}

To include phototherapy treatment in a toothbrush represents a novel approach of interest to daily oral care, since it has the potential to be a promising and cost efficient adjunctive tool for treating gingivitis and preventing periodontitis. This study has demonstrated that a toothbrush with an incorporated blue light LED emitting with an emission maximum at $450 \mathrm{~nm}$ was not able to give a sufficient significant adjunctive effect to toothbrushing with regard to reduction in dental plaque and gingival inflammation as well as in immunological inflammatory markers. Still, our suggestion is that antimicrobial blue light incorporated in a toothbrush could be beneficial if a $405 \mathrm{~nm}$ LED is used to obtain optimal phototherapeutic effect from a LED toothbrush. In future studies, the phototherapeutic of $405 \mathrm{~nm}$ LED toothbrushes should be investigated.

\section{Acknowledgements}

The Swedish Research Council, Contract No K2014-70X-22533-01-3, Praktikertjänst AB, Karolinska Institutet, Huddinge, Sweden and Stockholm Universi- 
ty, Stockholm, Sweden supported the study. We thank Timothy Lawrence at TJL Enterprises, Long Beach, CA, USA and Michael Barns at TechLight Systems, Ocala, FL, USA for supplying the blue light toothbrushes used in this study. The authors report no conflicts of interest. We thank Daniela Bureik, Department of Dental Medicine, Division of Periodontology, Karolinska Institutet, Huddinge, Sweden, for technical laboratory assistance in the analysis of inflammatory markers.

\section{Conflicts of Interest}

The authors declare no conflicts of interest regarding the publication of this paper.

\section{References}

[1] Etiology and Pathogenesis of Periodontal Diseases. https://www.ncbi.nlm.nih.gov/pubmed/15978238

[2] Albandar, J.M. (2005) Epidemiology and Risk Factors of Periodontal Diseases. Dental Clinics of North America, 49, 517-532. https://doi.org/10.1016/j.cden.2005.03.003

[3] Jin, L.J., Armitage, G.C., Klinge, B., Lang, N.P., Tonetti, M., et al. (2011) Global Oral Health Inequalities: Task Group-Periodontal Disease. Advances in Dental Research, 23, 221-226. https://doi.org/10.1177/0022034511402080

[4] Marsh, P.D., Head, D.A. and Devine, D.A. (2015) Dental Plaque as a Biofilm and a Microbial Community-Implications for Treatment. Journal of Oral Biosciences, 57, 185-191. https://doi.org/10.1016/j.job.2015.08.002

[5] Chapple, I.L., Van der Weijden, F., Doerfer, C., Herrera, D., Shapira, L., et al. (2015) Primary Prevention of Periodontitis: Managing Gingivitis. Journal of Clinical Periodontolgy, 42, S71-S76. https://doi.org/10.1111/jcpe.12366

[6] Sheiham, A. (1997) Is the Chemical Prevention of Gingivitis Necessary to Prevent Severe Periodontitis? Periodontology, 15, 15-24. https://doi.org/10.1111/j.1600-0757.1997.tb00100.x

[7] Albandar, J.M., Kingman, A., Brown, L.J. and Loe, H. (1998) Gingival Inflammation and Subgingival Calculus as Determinants of Disease Progression in Early-Onset Periodontitis. Journal of Clinical Periodontology, 25, 231-237. https://doi.org/10.1111/j.1600-051X.1998.tb02433.x

[8] Lang, N.P., Schatzle, M.A. and Loe, H. (2009) Gingivitis as a Risk Factor in Periodontal Disease. Journal of Clinical Periodontology, 36, 3-8. https://doi.org/10.1111/j.1600-051X.2009.01415.x

[9] Konig, K., Teschke, M., Sigusch, B., Glockmann, E., Eick, S., et al. (2000) Red Light Kills Bacteria via Photodynamic Action. Cellular and Molecular Biology (Noisy-leGrand, France), 46, 1297-1303.

[10] Maisch, T. (2007) Anti-Microbial Photodynamic Therapy: Useful in the Future? Lasers in Medical Science, 22, 83-91. https://doi.org/10.1007/s10103-006-0409-7

[11] Yang, L., Liu, Y., Wu, H., Song, Z., Høiby, N., et al. (2012) Combating Biofilms. FEMS Immunology and Medical Microbiology, 65, 146-157. https://doi.org/10.1111/j.1574-695X.2011.00858.x

[12] Soukos, N.S. and Goodson, J.M. (2011) Photodynamic Therapy in the Control of 
Oral Biofilms. Periodontology, 55, 143-166. https://doi.org/10.1111/j.1600-0757.2010.00346.x

[13] Kleinpenning, M.M., Smits, T., Frunt, M.H., van Erp, P.E., van de Kerkhof, P.C., et al. (2010) Clinical and Histological Effects of Blue Light on Normal Skin. Photodermatology, Photoimmunology and Photomedicine, 26, 16-21. https://doi.org/10.1111/j.1600-0781.2009.00474.x

[14] Oplander, C., Hidding, S., Werners, F.B., Born, M., Pallua, N., et al. (2011) Effects of Blue Light Irradiation on Human Dermal Fibroblasts. Journal of Photochemistry and Photobiology B: Biology, 103, 118-125. https://doi.org/10.1016/j.jphotobiol.2011.02.018

[15] Zhang, Y., Zhu, Y., Chen, J., Wang, Y., Sherwood, M.E., et al. (2016) Antimicrobial Blue Light Inactivation of Candida albicans. In Vitro and in Vivo Studies. Virulence, 7, 536-545. https://doi.org/10.1080/21505594.2016.1155015

[16] Ramakrishnan, P., Maclean, M., MacGregor, S.J., Anderson, J.G. and Grant, M.H. (2016) Cytotoxic Responses to $405 \mathrm{~nm}$ Light Exposure in Mammalian and Bacterial Cells: Involvement of Reactive Oxygen Species. Toxicology in Vitro, 33, 54-62. https://doi.org/10.1016/j.tiv.2016.02.011

[17] Fyrestam, J., Bjurshammar, N., Paulsson, E., Johannsen, A. and Östman, C. (2015) Determination of Porphyrins in Oral Bacteria by Liquid Chromatography Electrospray Ionization Tandem Mass Spectrometry. Analytical and Bioanalytical Chemistry, 407, 7013-7023. https://doi.org/10.1007/s00216-015-8864-2

[18] Fyrestam, J., Bjurshammar, N., Paulsson, E., Mansouri, N., Johannsen, A., et al. (2017) Influence of Culture Conditions on Porphyrin Production in Aggregatibacter actinomycetemcomitans and Porphyromonas Gingivalis. Photodiagnosis and Photodynamic Therapy, 17, 115-123. https://doi.org/10.1016/j.pdpdt.2016.11.001

[19] Soukos, N.S., Som, S., Abernethy, A.D., Ruggiero, K., Dunham, J., et al. (2005) Phototargeting Oral Black-Pigmented Bacteria. Antimicrobial Agents and Chemotherapy, 49, 1391-1396. https://doi.org/10.1128/AAC.49.4.1391-1396.2005

[20] Cieplik, F., Spath, A., Leibl, C., Gollmer, A., Regensburger, J., et al. (2014) Blue Light Kills Aggregatibacter actinomycetemcomitans Due to Its Endogenous Photosensitizers. Clinical Oral Investigations, 18, 1763-1769. https://doi.org/10.1007/s00784-013-1151-8

[21] Ashkenazi, H., Malik, Z., Harth, Y. and Nitzan, Y. (2003) Eradication of Propionibacterium acnes by Its Endogenic Porphyrins after Illumination with High Intensity Blue Light. FEMS Immunology and Medical Microbiology, 35, 17-24. https://doi.org/10.1111/j.1574-695X.2003.tb00644.x

[22] Maclean, M., MacGregor, S.J., Anderson, J.G. and Woolsey, G. (2009) Inactivation of Bacterial Pathogens Following Exposure to Light from a 405-Nanometer LightEmitting Diode Array. Applied Environmental Microbiology, 75, 1932-1937. https://doi.org/10.1128/AEM.01892-08

[23] Dai, T., Gupta, A., Murray, C.K., Vrahas, M.S., Tegos, G.P., et al. (2012) Blue Light for Infectious Diseases: Propionibacterium acnes, Helicobacter pylori, and Beyond? Drug Resistance Updates, 15, 223-236. https://doi.org/10.1016/j.drup.2012.07.001

[24] Hope, C.K., Hindley, J.A., Khan, Z., de Jong Ede, J. and Higham, S.M. (2013) Lethal Photosensitization of Porphyromonas gingivalis by Their Endogenous Porphyrins under Anaerobic Conditions: An in Vitro Study. Photodiagnosis and Photodynamic Therapy, 10, 677-682. https://doi.org/10.1016/j.pdpdt.2013.08.006

[25] Ahmed, I., Fang, Y., Lu, M., Yan, Q., El-Hussein, A.M.K., et al. (2017) Recent Patents on Light-Based Anti-Infective Approaches. Recent Patents on Anti-Infective 
Drug Discovery.

[26] Soukos, N.S., Stultz, J., Abernethy, A.D. and Goodson, J.M. (2015) Phototargeting Human Periodontal Pathogens in Vivo. Lasers in Medical Science, 30, 943-952. https://doi.org/10.1007/s10103-013-1497-9

[27] Genina, E.A., Titorenko, V.A., Belikov, A.V., Bashkatov, A.N. and Tuchin, V.V. (2015) Adjunctive Dental Therapy via Tooth Plaque Reduction and Gingivitis Treatment by Blue Light-Emitting Diodes Tooth Brushing. Journal of Biomedical Optics, 20, Article ID: 128004. https://doi.org/10.1117/1.JBO.20.12.128004

[28] Silness, J. and Loe, H. (1964) Periodontal Disease in Pregnancy. II. Correlation between Oral Hygiene and Periodontal Condition. Acta Odontologica Scandinavica, 22, 121-135. https://doi.org/10.3109/00016356408993968

[29] Loe, H. and Silness, J. (1963) Periodontal Disease in Pregnancy. I. Prevalence and Severity. Acta Odontologica Scandinavica, 21, 533-551. https://doi.org/10.3109/00016356309011240

[30] Directive, E. (2002) 95/EC "Restriction of Hazardous Substances in Electrical and Electronic Equipment” (RoHS Directive 2002).

[31] SP Technical Research Institute of Sweden AB (2010) Measurement of Blue LEDs on Toothbrush. SP Report MTkPX05459, 4 p. (In Swedish)

[32] Van der Weijden, G.A. and Hioe, K.P. (2005) A Systematic Review of the Effectiveness of Self-Performed Mechanical Plaque Removal in Adults with Gingivitis Using a Manual Toothbrush. Journal of Clinical Periodontology, 32, 214-228. https://doi.org/10.1111/j.1600-051X.2005.00795.x

[33] Sorsa, T., Mantyla, P., Tervahartiala, T., Pussinen, P.J., Gamonal, J., et al. (2011) MMP Activation in Diagnostics of Periodontitis and Systemic Inflammation. Journal of Clinical Periodontology, 38, 817-819. https://doi.org/10.1111/j.1600-051X.2011.01753.x

[34] Lee, A., Ghaname, C.B., Braun, T.M., Sugai, J.V., Teles, R.P., et al. (2012) Bacterial and Salivary Biomarkers Predict the Gingival Inflammatory Profile. Journal of Periodontology, 83, 79-89. https://doi.org/10.1902/jop.2011.110060

[35] Zhou, M., Meng, H.X., Zhao, Y.B. and Chen, Z.B. (2012) Changes of Four Proinflammatory Proteins in Whole Saliva during Experimental Gingivitis. The Chinese Journal of Dental Research: The Official Journal of the Scientific Section of the Chinese Stomatological Association (CSA), 15, 121-127.

[36] Syndergaard, B., Al-Sabbagh, M., Kryscio, R.J., Xi, J., Ding, X., et al. (2014) Salivary Biomarkers Associated with Gingivitis and Response to Therapy. Journal of Periodontology, 85, e295-e303. https://doi.org/10.1902/jop.2014.130696

[37] Boronat-Catala, M., Catala-Pizarro, M. and Bagan Sebastian, J.V. (2014) Salivary and Crevicular Fluid Interleukins in Gingivitis. Journal of Clinical and Experimental Dentitry, 6, e175-e179. https://doi.org/10.4317/jced.51403

[38] Zhang, J., Kashket, S. and Lingström, P. (2002) Evidence for the Early Onset of Gingival Inflammation Following Short Term Plaque Accumulation. Journal of Clinical Periodontology, 29, 1082-1085. https://doi.org/10.1034/j.1600-051X.2002.291206.x

[39] Murdoch, L.E., Maclean, M., Endarko, E., MacGregor, S.J. and Anderson, J.G. (2012) Bactericidal Effects of $405 \mathrm{~nm}$ Light Exposure Demonstrated by Inactivation of Escherichia, Salmonella, Shigella, Listeria, and Mycobacterium Species in Liquid Suspensions and on Exposed Surfaces. The Scientific World Journal, 2012, Article ID: 137805. https://doi.org/10.1100/2012/137805

[40] Maclean, M., McKenzie, K., Anderson, J.G., Gettinby, G. and MacGregor, S.J. 
(2014) $405 \mathrm{~nm}$ Light Technology for the Inactivation of Pathogens and Its Potential Role for Environmental Disinfection and Infection Control. The Journal of Hospital Infection, 88, 1-11. https://doi.org/10.1016/j.jhin.2014.06.004

[41] Fyrestam, J. and Ostman, C. (2017) Determination of Heme in Microorganisms Using HPLC-MS/MS and Cobalt (III) Protoporphyrin IX Inhibition of Heme Acquisition in Escherichia coli. Analytical and Bioanalytical Chemistry, 409, 6999-7010. https://doi.org/10.1007/s00216-017-0610-5

[42] Fyrestam, J. (2017) Escherichia Coli Is Killed by $405 \mathrm{~nm}$ Blue Light Due to Its Endogenous Porphyrins Induced by 5-Aminolevulinic Acid.

[43] Fukui, M., Yoshioka, M., Satomura, K., Nakanishi, H. and Nagayama, M. (2008) Specific-Wavelength Visible Light Irradiation Inhibits Bacterial Growth of Porphyromonas Gingivalis. Journal of Periodontal Research, 43, 174-178.

https://doi.org/10.1111/j.1600-0765.2007.01009.x

[44] McDonald, R., Macgregor, S.J., Anderson, J.G., Maclean, M. and Grant, M.H. (2011) Effect of 405-nm High-Intensity Narrow-Spectrum Light on Fibroblast-Populated Collagen Lattices: An in Vitro Model of Wound Healing. Journal of Biomedical Optics, 16, Article ID: 048003. https://doi.org/10.1117/1.3561903

[45] Kumar, A., Ghate, V., Kim, M.J., Zhou, W., Khoo, G.H., et al. (2016) Antibacterial Efficacy of 405, 460 and $520 \mathrm{~nm}$ Light Emitting Diodes on Lactobacillus plantarum, Staphylococcus aureus and Vibrio parahaemolyticus. Journal of Applied Microbiology, 120, 49-56. https://doi.org/10.1111/jam.12975 